XXXIX "Jaszowiec" International School and Conference on the Physics of Semiconductors, Krynica-Zdrój 2010

\title{
Excitation Mechanisms of CdTe/ZnTe Quantum Dots under Non-Resonant and Quasi-Resonant Regime
}

\author{
T. KazimierczUK ${ }^{a, *}$, J.A. Gaj ${ }^{a}$, A. Golnik ${ }^{a}$, M. GoryCA $^{a}$, M. NAWrocki $^{a}$,

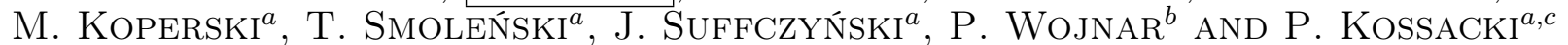 \\ ${ }^{a}$ Institute of Experimental Physics, Faculty of Physics, University of Warsaw, Hoża 69, 00-681 Warsaw, Poland \\ ${ }^{b}$ Institute of Physics, Polish Academy of Sciences, al. Lotników 32/46, 02-668 Warsaw, Poland \\ ${ }^{c}$ Grenoble High Magnetic Field Laboratory, CNRS, BP 166, 38042 Grenoble Cedex 9, France \\ We discuss possible mechanisms of quantum dot population control. A set of experiments, including \\ time-resolved photoluminescence, single photon correlations, excitation correlation, and photoluminescence \\ excitation is used to determine the actual mechanism under non-resonant and quasi-resonant regime.
}

PACS: 78.67.Hc, 78.55.Et

\section{Introduction}

An analogy between individual atoms and semiconductor quantum dots ("artificial atoms") is used since the beginning of the field [1]. This comparison points out the inherent property of quantum dots (QDs): 3D confining potential. The exact shape of this potential is in general difficult to study. However, in case of several lowest energy states, a satisfactory theoretical description can be achieved assuming a simplified case of harmonic potential in lateral dimensions and a separation of the in-plane effective mass Schrödinger equation from that along the growth axis [2]. This simple approach is sufficient to understand many different experimental results for QDs, from a simple shell-like emission pattern in the photoluminescence (PL) experiments [3] to elegant demonstrations of coherent light-matter interaction in single QDs [4].

The flexibility in possible confining potential shapes is not the only feature distinguishing QDs from real atoms. Another important difference lies in the presence of a host material surrounding each semiconductor QD. The role of the environment is crucial in QD excitation processes. Unfortunately, due to the complexity of a dot-environment system, a complete theoretical description of a real physical situation is hardly possible without additional assumptions. These difficulties are present, e.g., in case of excitation processes, where we are limited to statistical approach [5].

In this paper we review our experimental results on excitation mechanisms in $\mathrm{CdTe} / \mathrm{ZnTe} \mathrm{QDs}$ under two different regimes: non-resonant $\left(h \nu>E_{\mathrm{g}}\right)$ or quasi-resonant $\left(h \nu<E_{\mathrm{g}}\right)$ one, $E_{\mathrm{g}}$ being the barrier energy gap.

\section{Samples and experiment}

The experiments were performed on samples containing CdTe QDs embedded in ZnTe matrix. Two sam-

* corresponding author; e-mail: Tomasz.Kazimierczuk@fuw.edu.pl ple growth procedures were used. The first studies on non-resonant excitation mechanisms were performed on dots formed with growth-interrupt method [6]. More advanced experiments under non-resonant and quasi-resonant regime were done on dots grown with amorphous tellurium technique described in Ref. [7]. QDs grown with these two techniques were characterized with different depth of confining potential. QDs grown with growth-interrupt method confined only one orbital for electrons and holes while in case of dots grown with amorphous tellurium technique also higher excited states were observed.

Excitation mechanisms of the quantum dots were studied through their photoluminescence (PL) properties. Depending on the experiment, the samples were excited either with a frequency-doubled Ti:sapphire femtosecond laser or with a tunable cw dye laser. The studied sample was placed together with a specially designed immersion microscope [8] inside a pumped He-bath cryostat at 1.5-2 K. Light emitted by the excited sample was resolved with a monochromator and recorded with a CCD camera. The microscope objective allowed us to focus the laser beam to a spot below $0.5 \mu \mathrm{m}$ in diameter, which was sufficient to spectrally select emission lines originating from individual QDs.

In case of single photon correlations a Hanbury-Brown and Twiss (HBT)-type detection scheme [9] was used. In this case, the emitted light was split on a 50/50 beamsplitter and detected by a pair of monochromators equipped with avalanche photodiodes (APDs). This kind of detection scheme was particularly useful for identification of emission lines in the PL spectrum. The use of two separate detectors is necessary to circumvent a problem of a delay caused by the dead time inherent to APD detectors. Detailed description of the experiment is given in Ref. [10].

Due to the random character of self-assembled growth, substantial differences in PL spectra of different QDs were observed. For more detailed studies dots exhibiting most frequently observed emission pattern (Fig. 1) were selected. Typical PL spectrum of our dots include lines 


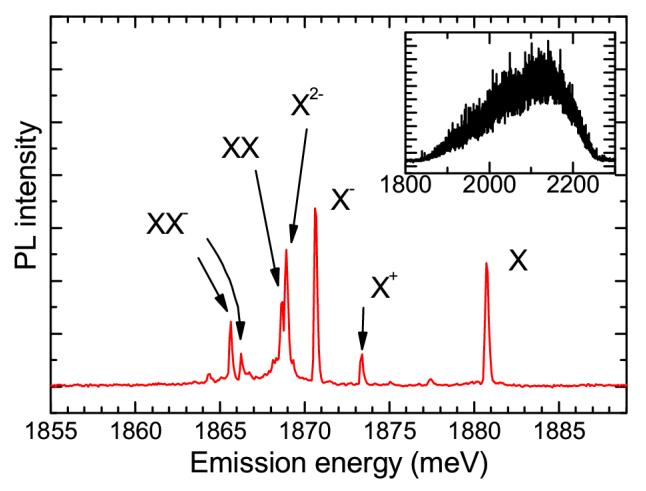

Fig. 1. Typical PL pattern of a single CdTe QD. The inset presents emission from ensemble of such QDs.

related to recombination of neutral exciton $(\mathrm{X})$, charged excitons $\left(\mathrm{X}^{+}, \mathrm{X}^{-}\right.$, and $\mathrm{X}^{2-}$ in more negatively charged dots), and neutral biexciton (XX). Differences in recombination energies of the observed excitonic complexes cannot be understood within the single-particle picture. They represent an indirect measure of the Coulomb interaction between confined carriers.

\section{Results and discussion}

\subsection{Non-resonant excitation}

In a photoluminescence experiment we observe light emitted by the sample excited with light of higher energy. In case of QDs, as well as other semiconductor nanostructures, the generic non-resonant excitation approach is widely used. In this scenario, the samples are excited with photons of energies higher than the energy gap of the barrier material. A detailed knowledge of the QD environment is hardly accessible and therefore theoretical models are not sufficient to provide quantitative predictions regarding detailed excitation mechanism of QDs by photocreated carriers. More promising results were obtained using an experimental approach.

Several different time-resolved techniques were used to address this issue. The simplest one is a time resolved measurement of PL intensity after an excitation pulse. We performed such an experiment with APD as a single-photon detector. Fast electronics was recording a delay between a synchronization signal from femtosecond oscillator and the detection of a photon emitted by the $\mathrm{QD}$ after a given excitation pulse. A typical result of the experiment is shown in Fig. 2. The temporal profile of the measured signal has asymmetric sawtooth-shape evidencing different timescales of involved processes: fast excitation of the QD after the pulse and relatively slow radiative decay. Experimentally determined radiative lifetime varied in range $200-500$ ps depending on the emission line; for different transitions in the same QD certain regularities between respective lifetimes were observed [11]. The rise time of the signal in Fig. 2 is comparable with the experimental resolution of $50 \mathrm{ps}$. Any faster processes are out of reach of this measurement.

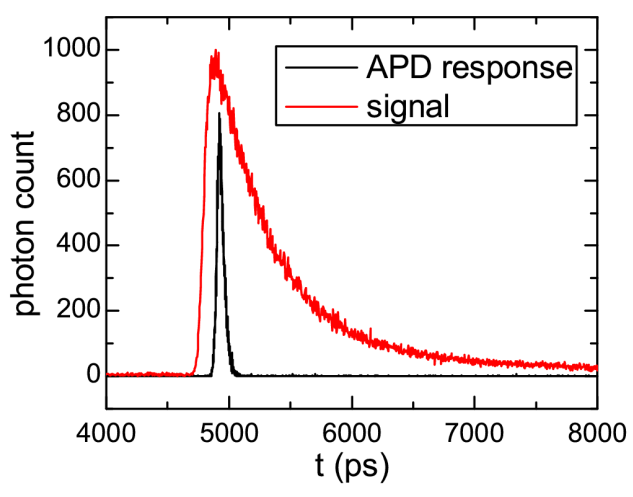

Fig. 2. PL dynamics of QD ensemble after a single excitation pulse. Response function was shifted horizontally for clarity.

To reach a deeper understanding of the mechanism of non-resonant excitation more advanced techniques are required, such as single photon correlations in the previously described HBT setup. A summary of the correlation measurement results is presented in Fig. 3. The observed variation in the photon pair count for small delays ( $\Delta t=0, \pm 1, \pm 2$ repetition periods) is related to the mechanism of QD population. Antibunching, i.e. strong suppression of the central peak $(\Delta t=0)$, indicates single photon emission from the studied QD and is independent of the details of the excitation process. On the other hand, intensities of peaks at $\Delta t= \pm 1$ are direct measure of probability of capturing a given number of carriers during a single excitation pulse. For example, in case of $\mathrm{X}^{-}-\mathrm{X}$ cross-correlation peak at $\Delta t=-1$ represents events of recombination of charged exciton (made of 2 electrons and 1 hole) provided that the previous excitation pulse resulted in recombination of neutral exciton (i.e. dot was left empty). The measured probability of such events is much lower than the probability of capturing a single carrier, which is encoded in $\Delta t=1$ peak. This asymmetry represents an evidence that the main excitation mechanism in the non-resonant regime is single carrier capture (as opposed to, e.g., capture of $\mathrm{e}-\mathrm{h}$ pairs). This conclusion was further verified quantitatively by construction of a rate equation model described in Ref. [10], which is presented schematically in Fig. 4.

Finally, the dynamics of the single carrier capture was studied in the excitation correlation measurements. The experiment based on time-integrated detection with CCD camera, similar to the case of basic PL measurement. Access to the dynamics was obtained by exciting the sample by pairs of pulses instead of single ones. The pulse pairs were produced in a Michelson-type interferometer, which allowed us to vary the delay between the two pulses. The measured time-integrated PL intensity as a function of inter-pulse delay is shown in Fig. 5a. The data reveal presence of a peak or dip in the PL signal for pulse delays in the range of tens of picoseconds. The sign of this feature was correlated with the charge state of the 

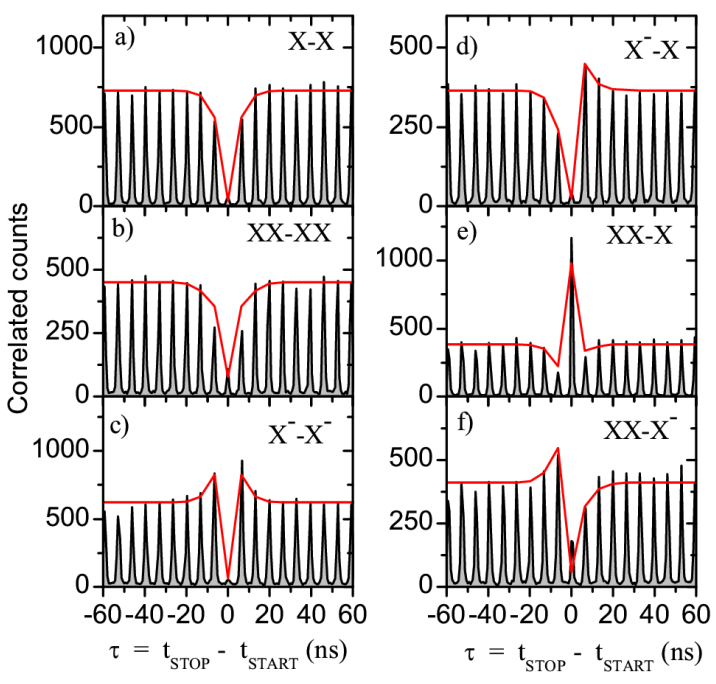

Fig. 3. Set of correlation histograms measured for a single QD. Solid lines represent fits of respective rate equation model (after Ref. [10]).

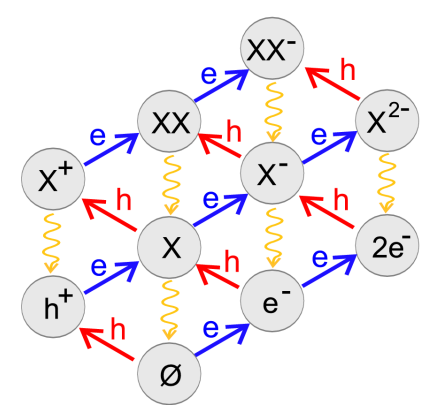

Fig. 4. Schematic representation of rate-equation model describing excitation dynamics of CdTe QDs. Letters "e" and "h" denote single carrier capture (electron and hole, respectively).

QD: average QD charge state was more negative for short delays. The feature was recognized as an effect of different trapping dynamics of electrons and holes [12]. We assumed that the asymmetry between carriers of different signs can be modelled as a difference in the values of carrier capture decay time between electrons and holes. We introduced this assumption into the rate equation model developed earlier to describe single photon correlation measurements. We found that the model calculations (Fig. 5b) satisfactorily reproduce the experimental observations. By comparing the simulation with the experimental data we identified an empirical timescale of $30 \mathrm{ps}$ as a characteristic capture time of electrons into the dot. The simulations indicate also that the characteristic capture time of holes is significantly faster, however, the obtained results are insensitive to its exact value.

\subsection{Quasi-resonant excitation}

The findings described above are inherent to non-resonant regime with great number of photo-created car-
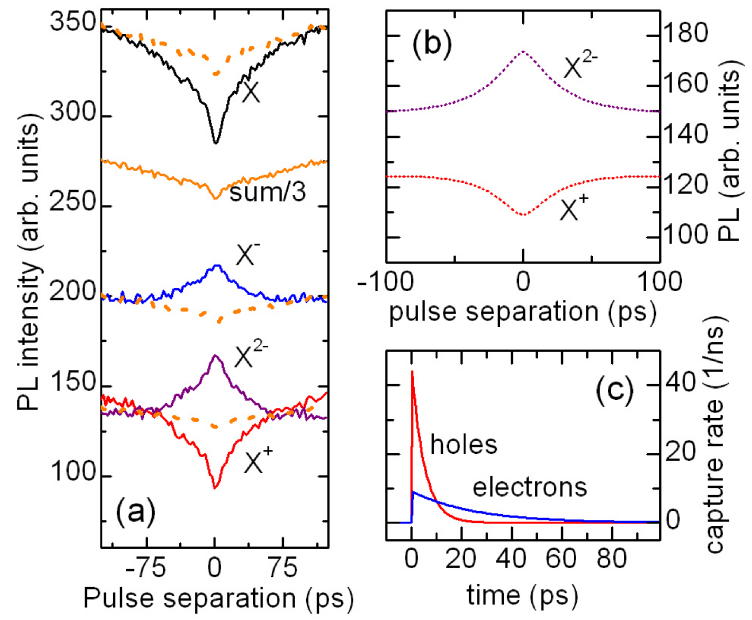

Fig. 5. (a) Results of excitation correlation experiment. Dotted lines follow total PL intensity of the QD and represent expected behavior basing on longer delays. (b) Profiles simulated within rate equation model assuming non-synchronous carrier capture. (c) Temporal profiles of carrier capture after an excitation pulse used in the simulation (after Ref. [12]).

riers in the surrounding host lattice. In principle, a completely different behavior can be expected in the quasi-resonant regime with excitation photon energy below energy gap of the barriers. In this case the light is no longer absorbed in the barrier but excitons bound to, e.g., QD or wetting layer are created directly. Therefore, in the case of quasi-resonant excitation the PL properties are expected to depend on actual excitation energy. Following this prediction, we performed photoluminescence excitation (PLE) experiments on our dots. During the experiments, we measured PL spectra while tuning $\mathrm{cw}$ dye laser in the range $2000-2200 \mathrm{meV}$. Typical results obtained for single CdTe/ZnTe QDs are presented in Fig. 6a. The measurement revealed presence of sharp resonances observed as an increase of QD PL intensity by an order of magnitude.

We performed a photon correlation experiment with a cw laser tuned to the resonance to compare quasi-resonant excitation mechanism with previously studied single carrier capture under non-resonant excitation. Representative results of this experiment are presented in Fig. 6c-e. Careful analysis of the measured histograms reveals that the dominant excitation mechanism is based on the QD population by introducing whole $\mathrm{e}-\mathrm{h}$ pairs into the dot instead of separate carriers. One of the consequences of excitation with $\mathrm{e}-\mathrm{h}$ pairs is relatively long timescale of random QD charge fluctuation. Indeed, the effects on the timescale of tens of nanosecond are clearly visible in the measured data (Fig. 6c-e). Photon pairs with shorter delay time are favoured to originate from the transitions in the same charge state. As a result, we observed an increased count of such events for auto-correlation (e.g. Fig. 6c) and cross-correlation between 

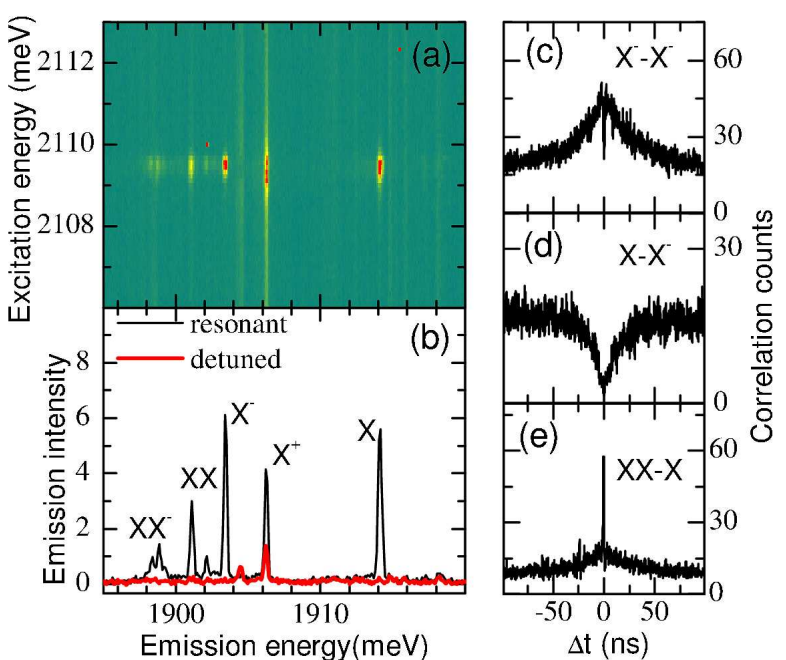

Fig. 6. (a) PLE and (b) PL of a single QD measured under resonant excitation (black line) and after detuning by $1 \mathrm{meV}$ (red line). Correlation histograms: (c) $\mathrm{X}^{-}-\mathrm{X}^{-}$autocorrelation, (d) anticorrelation between different charge states $\left(\mathrm{X}^{-} \mathrm{X}^{-}\right)$and $(\mathrm{e}) \mathrm{XX}-\mathrm{X}$ cascade $(\mathrm{af}-$ ter Ref. [13]).

lines of the same charge state (e.g. Fig. 6e), while a lower count was observed for cross-correlations between lines of different charge states (e.g. Fig. 6d).

The findings of the photon correlation experiments stay in agreement with the physical nature of the excitation resonance used in the measurement. Detailed studies of properties of these resonances show that the resonantly excited state corresponds to neutral exciton localized in a neighboring QD and that the coupling between the two dots leads to efficient excitation transfer between them [13]. Surprisingly, no higher excited states of the emitting QD have been identified in the PLE experiments. On the other hand, resonant excitation via a neighboring QD was frequently observed [14]. This finding reveals a common character of the interdot coupling in the studied self-assembled QD systems.

\section{Summary}

The examples presented above demonstrate two basic excitation mechanisms of the QDs: population by single carriers or by $\mathrm{e}-\mathrm{h}$ pairs. The first mechanism is dominant under non-resonant regime. More advanced experiments, such as excitation correlation spectroscopy, give us access to excitation processes in timescales shorter than excitonic lifetime. In case of our samples, we found characteristic time of electron capture as about 30 ps and significantly faster hole capture.
The second mechanism - excitation through $\mathrm{e}-\mathrm{h}$ pairs - is related mainly to the quasi-resonant regime. Coupled QD pairs spontaneously formed in our self-assembled systems provide a convenient opportunity to study such processes.

Given example of quasi-resonant excitation involving inter-dot energy transfer reveals shortages of idealized single dot model.

\section{Acknowledgments}

This work was partially supported by the Foundation for Polish Science, the Polish Ministry of Science and Higher Education as research grants in years 2010-2011. One of us (P.K.) acknowledges the support from European Project No. FP7/2007-2013-221515 (MOCNA).

\section{References}

[1] P.A. Maksym, T. Chakraborty, Phys. Rev. Lett. 65, 108 (1990).

[2] Single Quantum Dots: Fundamentals, Applications and New Concepts, Vol. 90 of Topics in Applied Physics, Ed. P. Michler, Springer-Verlag, Berlin 2003.

[3] A. Babinski, M. Potemski, S. Raymond, J. Lapointe, Z.R. Wasilewski, Phys. Rev. B 74, 155301 (2006).

[4] A.J. Ramsay, Semicond. Sci. Technol. 25, 103001 (2010).

[5] M. Grundmann, D. Bimberg, Phys. Rev. B 55, 9740 (1997).

[6] G. Karczewski, S. Mackowski, M. Kutrowski, T. Wojtowicz, J. Kossut, Appl. Phys. Lett. 74, 3011 (1999).

[7] F. Tinjod, B. Gilles, S. Moehl, K. Kheng, H. Mariette, Appl. Phys. Lett. 82, 4340 (2003).

[8] J. Jasny, J. Sepiol, T. Irngartinger, M. Traber, A. Renn, U. Wild, Rev. Sci. Instrum. 67, 1425 (1996).

[9] R. Hanbury-Brown, R.Q. Twiss, Nature 178, 1046 (1956).

[10] J. Suffczyński, T. Kazimierczuk, M. Goryca, B. Piechal, A. Trajnerowicz, K. Kowalik, P. Kossacki, A. Golnik, K.P. Korona, M. Nawrocki, G. Karczewski, J.A. Gaj, Phys. Rev. B 74, 085319 (2006).

[11] T. Smoleński, T. Kazimierczuk, M. Goryca, P. Kossacki, J.A. Gaj, P. Wojnar, K. Fronc, M. Korkusinski, P. Hawrylak, Acta Phys. Pol. A 119, 615 (2011).

[12] T. Kazimierczuk, M. Goryca, M. Koperski, A. Golnik, J.A. Gaj, M. Nawrocki, P. Wojnar, P. Kossacki, Phys. Rev. B 81, 155313 (2010).

[13] T. Kazimierczuk, J. Suffczyński, A. Golnik, J.A. Gaj, P. Kossacki, P. Wojnar, Phys. Rev. B 79, 153301 (2009).

[14] T. Kazimierczuk, A. Golnik, P. Wojnar, J.A. Gaj, P. Kossacki, Phys. Status Solidi B 247, 1409 (2010). 